\title{
PEANO DYNAMICS AS A MODEL FOR TURBULENCE AND STRANGE NONCHAOTIC BEHAVIOUR
}

\author{
M.S. EL NASCHIE \\ Cornell University, Ithaca, N.Y. 14853, USA \\ (Received March 15, 1991)
}

\begin{abstract}
The note outlines a scenario for a two-dimensional dynamic system to possess strange nonchaotic behaviour in the presence of two frequency quasi periodic forcing. Implications for turbulence are also discussed.
\end{abstract}

PACS numbers: 05.45. +b

\section{Preliminary remarks}

Recent investigations [1-4] revealed the existence of strange, yet nonchaotic attractors in quasi periodically driven oscillators. These are fractal attractors which look topologically similar to the more familiar strange attractors, yet possess negatve Liapunov exponents and are thus nonchaotic. Also recently O. Roessler et al. gave convincing reasons for anticipating new strange chaotic phenomena in four dimensions $[5,6]$. Now the simplest quasi periodically forced oscillators have a four-dimensional phase space. Considering that Ruelle, Takens and Newhouse envisage chaos as a sequence of finite number of Hopf bifurcations leading to a totally unstable torus in four dimensions [7] then it is understandable that one may be inclined to speculate on possible cross connections between all these different lines of thought.

In what follows we outline a scenario which is similar to a Smale horse shoe [7] and may serve as a prototype for strange, but nonchaotic behaviour. We show that the action of contracting, stretching and special form of twist-folding of the phase space in a way similar but not identical to the horse shoe, leads to a distinct form of dynamics. The invariant set of this dynamics are Cantor-like objects and may be shown to be oriented on a Peano curve-like discrete manifold [8]. The immediate consequence of this picture is that we may anticipate a Poincare map of a system 
with strange nonchaotic behaviour to have a fractal dimension, tending towards $d_{c}=2$. Finally we give some arguments showing that we may indeed expect new phenomena in four-variable systems [6].

\section{A fractal model for phase space deformation}

Consider first a very simple, Cantor set geometrical construction which gives by and large the main features of Smale's horse shoe dynamics [7]. The rule governing this construction is to delete the middle cross of a unit square which is formed from nine equal smaller squares. Removing from the remaining four squares again the middle cross and so on ad infinitum, it is easy show that the area of the resulting set tends towards zero while its Hausdorff dimension tends towards $d_{\mathrm{c}}=\log _{2} 4 / \log _{2} 3 \approx 2(0.6309) \approx 1.261$.

This is clearly the Cartesian product of two middle third Cantor sets. This set with zero Lebesgue measure [9-11] thus has the same capacity dimension as the Koch curve which is used frequently in modelling many natural objects [8]. Now, the four corner squares which ultimately give rise to this Cantor set do correspond to the invariant set of a two-dimensional horse shoe map and are analogous to the intersection areas of the horse shoe and its preimage as explained with considerable lucidity for instance in the book of Nicolis and Prigogine [7]. In fact, if the dissipation parameter of a one-dimensional Smale horse shoe is made equal to $\alpha=7.05595$ then the fractal dimension will equal that of our two-dimensional map, namely $d_{\mathrm{c}}=1.261$. Alternatively, one could choose $\alpha=3 / 2$ to reach the same result for the two-dimensional horse shoe. Returning now to phase space, it is very simple to see that dynamics which can be attached to this simplistic fractal construction is: pressing, streching and bending in a $\mathcal{C}$ shape; then repeating the procedure ad infinitum. Suppose now that in addition to these actions we introduce a periodic torsional (twist) deformation before bending into an $\mathcal{S}$ shape. It is not difficult to see that an idealized form of the projection of such a torsional deformation of a long stretched rectangular strip could look similar to the nine elements of the forty five degrees rotated square drawn on the original square of Fig. 1. This may also be demonstrated by folding a twisted long paper strip (see Fig. 2). It is an elementary matter to establish that the capacity of the resulting geometrical set as we repeat this specific deformation mechanism is $d_{\mathrm{c}}=\log 36 / \log 6=2$. In what follows we will attempt to make it plausible that the resulting Cantor set-like objects generated by this iteration are related to the dynamics of a Peano-like curve [7] as well as to quasi periodically forced two-dimensional maps and strange nonchaotic attractors.

\section{The discrete Peano curve - fat fractals}

In the following the original Peano curve, to use Mandelbrot's terminology [8], will serve as the basis of our discussion. For this form of the curve we need at least a grid of nine squares (Fig. 3). These squares define the forty five degrees rotated square shown in Fig. 3 whose Hausdorf dimension when iterated is easily 


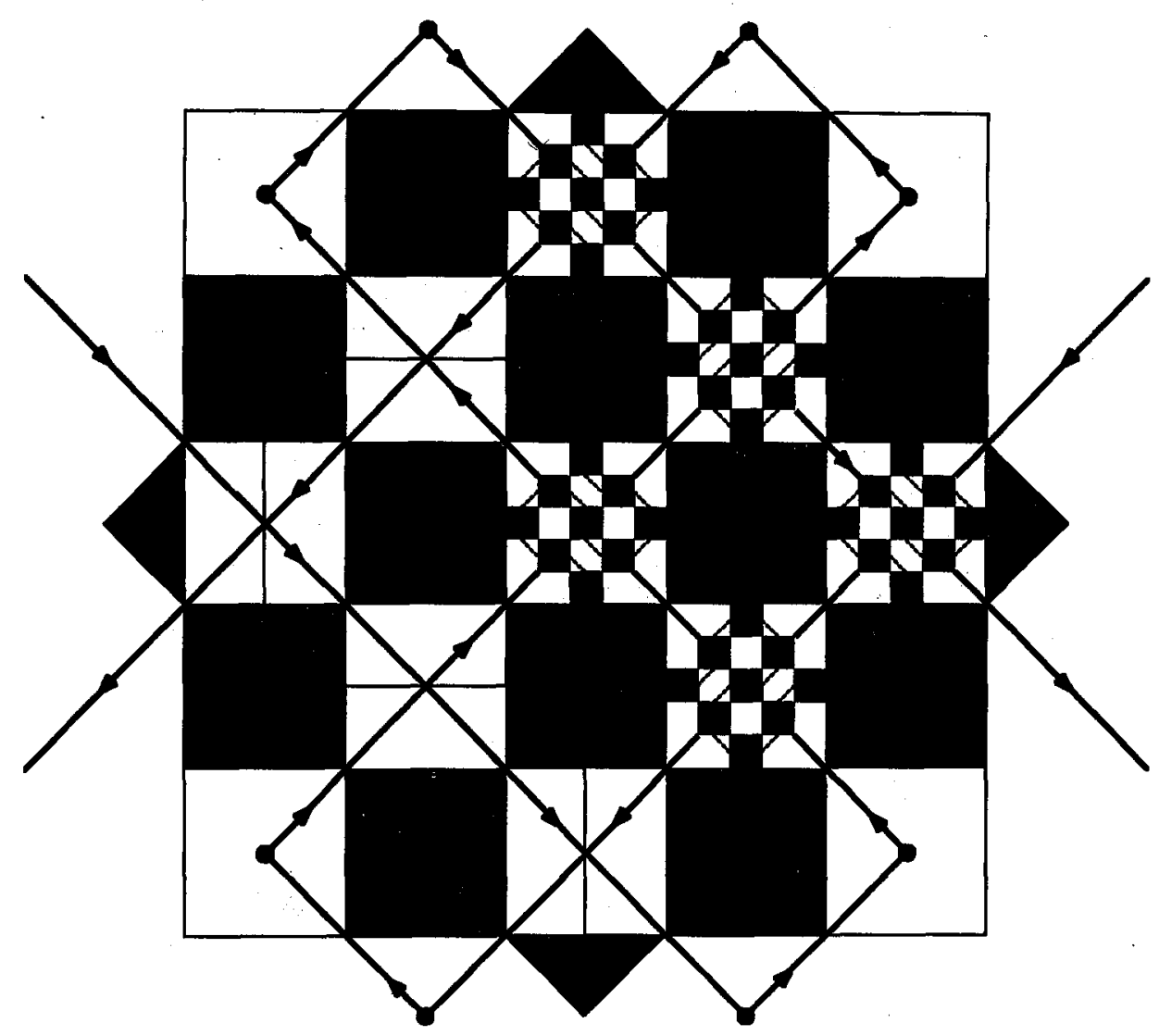

Fig. 1. A geometrical construction to explain the Peano dynamics iteration. Two squares can be seen. These are the five by five large square $\left(d_{c}=\log 13 / \log 5\right)$ and the forty degrees rotated square $\left(d_{c}=\log 36 / \log 6\right)$. In the first iteration only the yellow squares at the intersections of $\mathcal{S}$ and $\mathcal{S}^{-1}$ are retained. In the second iteration only the nine green squares are retained and so on, ad infinitum. 


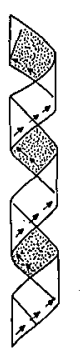

(a)

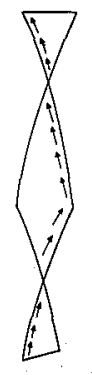

(b)

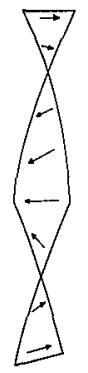

(c)

Fig. 2. Twist-folding of a paper strip into a helicoid shape. Note that such structures play an important role in many technical problems, such as microsurgical and electro-optical instruments.

shown in Ref. [8] to be 2. At the same time, the nine squares lie on two large Peano blocks as shown in Fig. 1. This curve, as it is well-known, possesses the properties of being area filling, selfavoiding and ergodic. Consequently its capacity dimension will tend towards $d_{c}=2$ and as will be reasoned later on in some detail, its information dimension will tend towards unity $[7,8]$. As we repeat our procedure in the sense of a discrete iterated map we see that each of the nine squares of all new sets of the small green squares lies on smaller and smaller Peano islands (Fig. 1) which we may now describe as discrete Peano curves. More precisely these curves have a double nature - they are globally discrete but locally connected, as can be seen from Fig. 1.

The capacity dimension remains nevertheless the same. Superficially, this might be regarded as logically inconsistent at least in the traditional geometrical sense, but again the notion of area-like curve is itself inconsistent in the same way. In Fig. 1 we see how the nine squares lie in a vertical larger square made of 25 squares. Finally the entire dynamics of an $\mathcal{S}$ form bending deformation of the pressed, stretched and twisted "phase space" lies in a seven by seven larger square. The invariant points of the set defining the starting point of the discrete Peano curve construction lies now on the intersections of the $\mathcal{S}$ deformed shape indicated in Fig. 1 with red lines and arrows and their pre-image $\mathcal{S}^{-1}$. A first estimation of the area left is $A \approx 0.6$ of the unit area but this will not be given here. It may be of interest however, to note that the critical probability of percolation in certain simulations considered for instance in Ref. [9] was found to be $P \approx 0.6$.

One may also note that the present picture of a discrete Peano map is reminiscent of fate fractals where we have an area coverage of a finite portion of the total initial area but with holes in it on all scales. For a middle third Cantor set for instance, if we delete $(1 / 3)^{n}$ for the $n$-th iteration we obtain a fate fractal Cantor set. However in this case we have $d_{\mathrm{c}}=1$ instead of $d_{\mathrm{c}}=0.6309$ and the remaining length is $\ell_{F}=0.56$ of the original unit length. In fact the same result was obtained by Farmer [11] for a two-dimensional system with quasi periodical orbits. He found 
that the fat fractal set takes about $A_{F} \approx 0.56$ of the unit area that represents the full phase space. This is close to $A \approx 0.6$ found here.

\section{Relation to quasi periodic forcing and four-dimensional phase space}

We have seen how a seemingly trivial change of symmetry due to an out of plane torsional movement radically changes the capacity-Hausdorff dimension of the geometrical configuration of an idealized phase space. This movement obviously mimic the action of quasi periodic forcing and the spiralling movement on a torus. Consequently our discrete map may be regarded in a sense as a quasi periodically forced horse shoe-like map. That might be the explanation for the numerically observed link between this form of forcing and the associated capacity dimension of possible strange attractors. Numerical calculations for these type of attractors have repeatedly shown for the Poincare maps a capacity dimension near to $d_{\mathrm{c}}=2$ as well as a positive Lebesgue measure [1-4]. In the case of a pendulum for instance, one finds for both

$$
\ddot{\phi}+0.005 \dot{\phi}+0.027 \sin \phi=0.2\left[\cos t+\cos \left(\frac{\sqrt{2}}{10} t\right)\right]
$$

and

$$
\ddot{\phi}+0.01 \dot{\phi}+0.0272222 \sin \phi=0.15\left[\sin t+\sin \left(\frac{\sqrt{2}}{10} t\right)\right] \sin \phi
$$

a strange nonchaotic attractor in the $\varphi-\dot{\varphi}$ Poincare map with a fractal capacity dimension

$$
d_{\mathrm{c}} \approx 1.81 \text { and } d_{\mathrm{c}} \approx 1.9042 \text {, }
$$

respectively. This alone may suggest that the present model correctly reflects a substantial part of the phenomenon of nonchaotic strange behaviour. One has, of course, to remember that twist in a two-dimensional horse shoe is not permissible which shows the crucial role played by the dimensionality of the phase space. In fact, for a four-dimensional phase space such as that of a quasi periodically forced pendulum one could argue that "typically" a Poincaré map projection would have a fractal dimension $d_{c}=2$ regardless of whether the attractor is chaotic or nonchaotic. This may be shown analytically by rescalling the phase dimension using an appropriate measure.

The starting point of this analysis is the generally accepted realization that fractals [10] are the carries of complex strange behaviour. Second, we follow Yorke's conjecture that single Cantor sets are somehow the back bone of all strange behaviour [1]. To that we add what is intuitively evident namely that in one dimension the simplest fractal set is Cantor's middle third set [12] with $d_{c}=\log 2 / \log 3$. If we accept this, then we can claim that in four-dimensional phase space a strange set will typically have a Cantor-like fractal dimension $d_{c} \approx 4$. This result is reached 

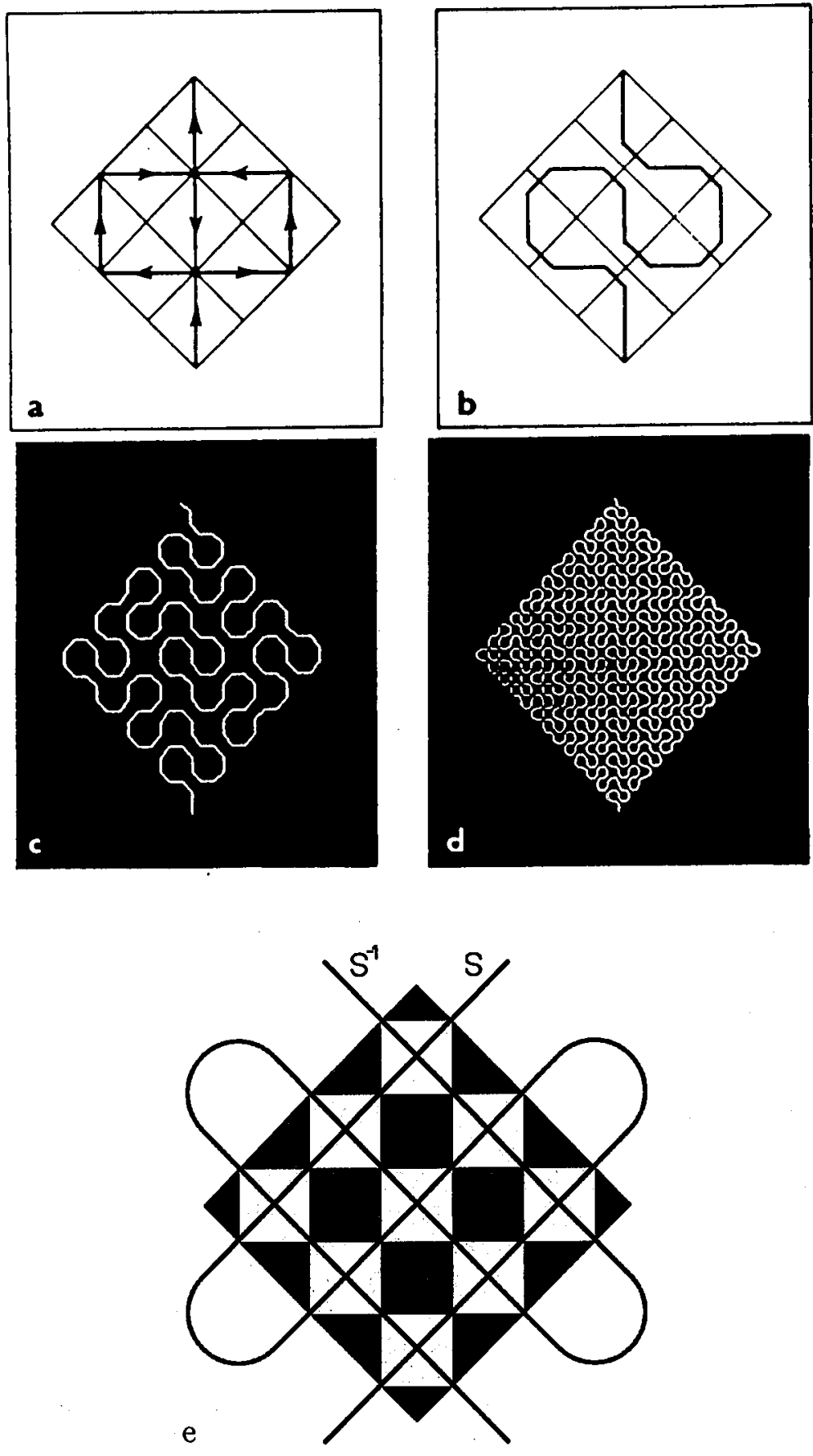

Fig. 3. The construction of a piano area filling curve $\left(d_{c}=\log 9 / \log 3\right)(a-d)$. The $\mathcal{S}$ and $\mathcal{S}^{-1}$ intersections defining the invariant set for the first iteration (e). 
using the following scaling argument. The idea is to find the equivalent to a triadic Cantor set in two dimensions. Such a set should be triadic Cantorian in every conceivable direction. It cannot therefore be the Cartesian product of two such sets, $d_{\mathrm{c}}=\log 4 / \log 2$ nor a Cantor target $d_{\mathrm{c}}=1+\log 2 / \log 3$. However, we know that a unit area $A$ of an Euclidian manifold is given by $A=(1)(1)=1$ and consequently a corresponding quasi area of a Cantor set is $A_{c}=\left(d_{c}\right)\left(d_{c}\right)$. It follows then that in order to normalize $A_{\mathrm{c}}$ it must be multiplied by the normalization factor $e_{2}=\left(A / A_{c}\right)_{2}$. By analogy in $n$ dimensions we would have $e_{n}=\left(A / A_{c}\right)_{n}$. Denoting the $n$-th Cantor-like fractal dimension in $n$-dimensional space by $d_{\mathrm{c}}^{(n)}$ and the dimension of the corresponding Euclidian space in $n$ dimensions by $d_{\mathrm{E}}^{(n)}=n$ it follows then that

$$
d_{c}^{(n)}=e_{n} d_{c}=d_{c} /\left(d_{c}\right)^{n}=\left(1 / d_{c}\right)^{n-1}=\left(d_{s}\right)^{n-1},
$$

where $d_{\mathrm{s}}$ is termed the escalation factor. This is the set which we are looking for and the result is now evaluated for $d_{c}=\log 2 / \log 3$ in Table I, where we have introduced a new quantity termed co-dimension defined as $c=d_{\mathrm{E}}^{(n)}-d_{\mathrm{c}}^{(n)}$. Note that $d_{\mathrm{s}}$ could be equally interpreted as the Floquet multiplier of a discrete map

$$
d_{(m+1)}=d_{(m)} / d^{(o)},
$$

where $n=m+1, d^{(o)}=d_{c}^{(o)}$ and $d_{\mathrm{s}}=\partial\left(d_{(m+1)}\right) / \partial\left(d_{(m)}\right)=1 / d^{(o)}$.

TABLE I

\begin{tabular}{l|c|c|c}
\hline \hline & $d_{\mathrm{E}}^{(n)}$ & $d_{\mathrm{c}}^{(n)}$ & $C$ \\
\hline Basic assumption & 0 & $d=0.63092$ & $C=d_{\mathrm{E}}^{(n)}-d_{\mathrm{c}}^{(n)}$ \\
\hline Normality & 1 & 1 & - \\
\hline & 2 & 1.58496 & 0.41504 \\
& 3 & 2.51210 & 0.48790 \\
Results & 4 & $\underline{3.98159}$ & 0.01841 \\
& 5 & 6.31067 & -1.31067 \\
& 6 & 10.00218 & -4.00218 \\
& 7 & 15.85309 & -8.85309 \\
& 8 & 25.12655 & -17.12655
\end{tabular}

There are a few interesting obserwations here. First $d_{c}^{(n+1)} / d_{c}^{(n)}=d_{s}$ is the fractal dimension of the Sierpinski gasket which is the prototype of fractal lattices with infinite hierarchy of semi-loops. Second, for all $n<4$ we have $n>d_{\mathrm{c}}^{(n)}$ while for $n>4$ we have $d_{\mathrm{c}}^{(n)} \gg n$. Only at $n=4$ we have a Cantor-like structure which comes very near to a space filling set. The two-dimensional geometrical analogue of this is the Peano curve which as mentioned ealier is ergodic and shares a few properties with fat fractals $[11,13]$. We may say therefore that at $n=4$ the set is almost ergodic. This point is clearly marked by the co-dimension $C$ becoming very small and then abruptly changing its sign to negative as can be seen in Table I.

It follows then that for a system with four-dimensional phase space $(n=4)$, a two-dimensional Poincare section will typically have a capacity dimension

$$
d_{\mathrm{c}} \rightarrow \sim[(\sim 4)-(\sim 2)] \rightarrow \sim 2 .
$$


Here we have assumed that the map is very weakly dependent on the phase of forcing. A numerical justification of this independence may be found in Table 6-2 of Ref. [19]. The paradoxal fact that the fractal dimension becomes substantially larger than the phase space dimension for $n=4$ is a clear indication of a very rugged hypersurface of possible strange attractors and may be related to what has been predicted by Roessler. It also indicates that we will have self intersections and complete loops instead of the semi-loops and selfavoidence of the Sierpinski gasket and the Peano curve.

\section{Insensitivity to initial conditions}

So far we have not explained why the just described Peano dynamics is nonchaotic. Leaving the ergodicity of the curve aside, a possible mechanism which may be at least a partial explanation is also related to the spiralling twist movement of the two frequencies, quasi-periodic forcing. The horse shoe deformation have all the basic elements of intuitive thinking, namely stretching, contraction and bending. To that we add now torsional twisting. Taking a long strip of paper of the length $\ell$ and width $a$ where $\ell \gg a$, we may start by dividing the strip in $n$ parts using a pencil so that $(\ell / n) / a=1$. Now we fold only every second of the drawn squares along the diagonal in one direction only. Subsequently one cuts out all parts that overlap, except for a very thin seam to keep the chain connected. The result is a long chain identical to one row of the rotated Peano squares of Fig. 1. We could have arrived at the same form by twisting the paper strip in many waves like a long helical spring then flattening it simply by pressing it on an even surface. The most important, though trivial observation here is that the length of the paper strip will now be reduced. For $\ell \rightarrow \infty$ the reduction will tend to $50 \%$. This percentage is, of course, dependent on the ratio $(\ell / n) / a$ which is analogous to the frequency of quasi periodic forcing. The shortening is clearly in the opposite direction to the axial stretching of the map. It follows then that analogous to one-dimensional horse shoe maps, the Liapunov exponents may be written as

$$
\lambda_{1}=\ln (\gamma-\beta) ; \quad \lambda_{2}=-\ln \alpha \gamma,
$$

where $\gamma$ is the factor of stretching in one direction, $\alpha$ is the factor of compression in the perpendicular direction while $\beta$ is the factor of compression produced by the shortening due to torsional waves. Nonchaotic behaviour would consequently be associated with dynamics for which we have $0 \leq(\gamma-\beta)<1$. It is very important to observe here that a shortening factor $\beta$ which is on its own sufficient to just make $\lambda_{1}$ smaller than zero, corresponds to a deformation of the Peano squares to rectangulars. This in turn causes the fractal dimension to drop under 2. For a rectangular with $a / b=2$ for instance, we obtain $d_{c}=\log 32 / \log 6 \approx 1.93$. We feel therefore that due to inaccuracy and difficulties of calculating $d_{c}$ it is not easy to distinguish between chaotic and nonchaotic attractors based upon this criterion alone when $d_{\mathrm{c}}$ is very close to $d_{\mathrm{c}}=2$. However we anticipate that in general nonchaotic attractors will have $d_{\mathfrak{c}} \leq 2$ in addition to an information dimension $d_{\mathrm{I}} \geq 1$. 


\section{Another view of the problem - the Kaplan-Yorke conjecture}

The nonchaoticness of our map can be argued in a different more subtle, but also slightly terse way. To do this we appeal to two facts and an established conjecture. First we know that ergodicity in all its different definitions will always guarantee that the orbit must cover the energy surface uniformly. It follows then that the pointwise dimension will be equal to its smooth Euclid manifold which in our case is two. Second, we know that the information dimension which is not a simple matric-dimension, implies that the information entropy $\mathcal{H}(\varepsilon)$ is an average of $N(1 / \varepsilon)$ of the pointwise dimension. Combining these two facts for the "discrete" Peano dynamics of our map it follows that $d_{\mathrm{I}} \approx \frac{1}{2} d_{\mathrm{P}} \approx 1$ for two dimensions. An intuitive explanation of this may be found by looking to the problem as being analogous to fat fractals. Using Kaplan-Yorke conjecture about the equality of Liapunov dimension and the information dimension, it is a relatively simple matter to show that that $d_{\mathrm{I}}=1$ implies Liapunov exponents combination guaranteeing insensitivity to initial conditions and thus nonchaoticness.

\section{Discussion and conclusion}

Relatively recently S. Donaldson made the discovery of a non-standard smooth structure on 4-space. He found that Lie groups of all rotations is simple for all dimensions higher or lower than 4 but not 4 . In phase space of dynamical systems four dimensions also seem to be, associated with strange nonchaotic attractors, wrinkled attractors and other interesting phenomena as pointed out by $O$. Roessler. We have speculated on the relevance of some of the numbers displayed in Table I in particular $n=d_{c}^{(n)}=4$. This may be interpreted in some intuitive sense as suggesting that the hosting four-dimensional Euclidian phase space of a possible strange attractor is typically saturated with fractals. In turn this meant that it is ergodic and eventually fill the entire phase space. Consequently the Poincare map is also area filling. This means $d_{c} \rightarrow 2$ and quite frequently also $d_{\mathrm{I}} \rightarrow 1$. This could provide some intuitive basis for Ruelle, Takens and Newhouse theorem since three-dimensional torus implies at least four-dimensional phase space [20]. According to $n=d_{c}^{(n)}=4$ this is now a highly critical state because any further increase in dimension say $n=5$, would ob viously lead to self intersection replacing the semi-loops of the Sierpiński construction by homoclinic loop soliton and even cusps as a homotopic limit for the loops because theoretically and paradoxically the fractal dimension $d_{\mathrm{c}}^{(5)} \approx 6.3$ is significantly higher than $n=5$.

A further interesting observation regarding Table $I$ is that for any three

successive dimensions $d_{c}^{(n)} \approx d_{c}^{(n-1)}+d_{c}^{(n-2)}$. This is strongly reminiscent of the Fibonacci numbers [4] and the corresponding dimension will be termed the Fibonacci fractal dimension. Should we insist that $d^{(n)}=d^{(n-1)}+d^{(n-2)}$ then we find that at $n=4$ the corresponding Cantor-like dimension is $d_{c}=4.23606$ while the Sierpiński gasket [13] is replaced by $d_{\mathrm{c}}=1 / \phi=d_{\mathrm{s}}$ where $\phi$ is the golden mean [12]. In fact our Table I becomes identical to the table calculated by Cook [14] for Botticelli's Venus. It might be intersting at this point to determine the escalation 
value $d_{\mathrm{s}}$ corresponding to exact critical equality of $d_{\mathrm{s}}^{(n)}$ and $n$ in four dimensions. This is an elementary application of our formula relating $d_{c}^{(n)}$ to $n$. This way one finds

$$
\left(1 / d_{c}^{(n)}\right)^{n-1}=\left(d_{s}\right)^{n-1}=n ; \quad\left(1 / d_{c}^{(4)}\right)^{4-1}=d_{s}^{4-1} ; \quad d_{s}=\sqrt[3]{4} \approx 1.587 .
$$

This is very close to the Sierpiniski gasket [18] $d_{\mathrm{c}}=1.58496$. Now a single Cantor set is easily made to have any fractal dimension between one and zero. Within this range it is now interesting to consider the consequence of having taken a Cantor set with Hausdorff dimension $d_{c}=\log 2 / \log 4$. In this case $d_{c}=0.5$ seems to be a distinct value between one and zero which may be regarded naively as the most "fractal" value in this unit interval. It is also the correlation dimension found for period doubling chaos in the one-dimensional logistic map as well as the probability describing the random behaviour of the tent map. It is an elementary matter to show, using the same previous formula, that $d_{c}=2$. The critical state thus shifts from $n=4$ to $n=2$. This is however another way of viewing the "critical" ergodic state $n=4$ in case of quasi periodically forced horse shoe maps displaying Peano-like dynamics [15]. Finally let us consider the implication of shifting criticality in the present ergodic sense to $n=3$. This clearly implies an escalation factor $d_{\mathrm{s}}=\sqrt{3}=1.732050$. Notice that in this case $d_{\mathrm{c}}^{(2)}=1.73205$ is indeed a value found frequently in two-dimensional Poincaré maps of dynamic systems as well as numerous fractal objects found in nature [13]. The role of multifractals as well as fractal sets made up of the union of different fractal subsets in developing more accurate mathematical models have not been discussed here. Looking again at Table I. One may speculate if fully developed turbulence has a fractal dimension $d_{c} \approx 6.3$ and that five-dimensional phase space is required to study this phenomenon. This would be for instance a nonlinearly oscillating set described by a phase space $x, \dot{x}$ and $x^{\prime}$ representing temporal and spatial oscillation of a state variable $x$. In addition we need a spatial fluctuation $w_{x}$ and a temporal fluctuation $w_{t}$ as forcing frequencies. This makes them indeed five variables. Another worthwhile observation is that the Fibonacci fractal dimension $d_{\mathrm{F}}^{(3)}=1+1 /(\log 2 / \log 3)=2.584496$ is identical to $d_{c}=1 /(\log 2 / \log 6)$, where $\log 2 / \log 6$ is clearly a reasonable measure of the fractal dimension at period 3 chaos of a Feigenbaum cascade. Note also that $d_{\mathrm{c}}=\log 2 / \log 6=0.387$ is very close to the smallest value $(d=0.378)$ found for period 3 chaos of the logistic map $[16,17]$.

A final point which is worth further careful investigations is the possible connection between loop soliton and chaos found recently in connection with diffusion-like process [18] and the Sierpiński gasket structure which we hinted at earlier on. The preliminary generalization of this structure to higher dimensions has lead to the fractal dimension $d_{\mathrm{c}}=\log (s+1) / \log 2$ where $s=3,4, \ldots$. This means that we need a seven-dimensional space for $d_{c}=3$ and that the co-dimension will never converge to zero. Although this is a negative result, the fact that for three and seven dimensions we have an integer as a fractal dimension is worth noting.

It is, of course, important to be cautious and appreciate the role of the 
proximity of some rational and irrational numbers such as $3 / 4, \pi / 5, d_{\mathrm{c}}=(1+\sqrt{5}) / 2$ and $d_{\mathrm{c}}=\log 2 / \log 3$, in arriving at some of the preceding conclusions.

We have made our case that the $\phi-\dot{\phi}$ Poincaré map capacity dimension of strange nonchaotic attractors of a quasi periodically, two frequencies forced system is $d_{\mathrm{c}}=2$ using different arguments. Taken on their own, none of these arguments is completely conclusive. However, taken altogether, we feel that they converge towards an intuitive informal proof.

\section{References}

[1] C. Grebogi, E. Ott, S. Plikand, J. Yorke Physica D 13, 261 (1984).

[2] F.J. Romeizas, E. O.tt, Phys. Rev. A 35, 4404 (1987).

[3] A. Bondeson, E. Ott, T. Antonsen, Phys. Rev. Lett. 55, 2103 (1985).

[4] M.S. El Naschie, in: Proc. ICIAM 1991; in: Proc. CANCAM 1991 p. 756.

[5] O.E. Roessler, J.L. Hudson, in: Chaotic Dynamics in Brain Function, ed. E. Basar, Springer, Berlin 1989, p. 113.

[6] O.E. Roessler, J.L. Hudson, M. Klein, C. Mira, in: Nonlinear Dynamics in Engineering Systems, ed. W. Schiehlen, Springer, Berlin 1990, p. 265.

[7] G. Nicolis, I. Prigogine, Die Erforschung des Komplexen, Piper, München 1987.

[8] B. Mandelbrot, The Fractal Geometry of Nature, W.H. Freeman, New York 1983.

[9] R. Durrett, Lecture Notes on Particle Systems and Percolation, Wadsworth, California 1988.

[10] K.H. Becker, M. Dorfler, Dynamical System, Cambrige Press, Cambridge 1989.

[11] S. Eubank, D. Farmer, in: 1989 Lectures in Complex Systems, ed. E. Jen, Addison Wesley, Redwood City 1989, p. 75.

[12] I. Stewart, Does God Play Dice?, Penguin, London 1989.

[13] T. Vicsek Fractal Growth Phenomena, World Scientific, Singapore 1989.

[14] T.A. Cook, The Curves of Life, Dover Publications, New York 1989, reprint of original edition published by Constable and Company, London 1914.

[15] M.S. El Naschie, to be published in Specul. Sci. Technol. (1991).

[16] S. Grossmann, in: Ordnung und Chaos, ed. W. Gerok, S. Hirzel Wissenschaftlicher Verlag, Stuttgart 1989, p. 101.

[17] M.S. El Naschie, Stress, Stability and Chaos, McGraw Hill, London 1990.

[18] M.S. El Naschie, Acta Phys. Pol. A79, 757 (1991).

[19] F. Moon, Chaotic Vibration, J. Wiley, New York 1987.

[20] D. Rulle, F. Takens, Commun. Math. Phys. 20, 167 (1971). 